


\section{GELÄNDER FÜR DIE DURCHQUERUNG EINES UNSICHEREN TERRAINS. Neue Publikationen zu Theorie und Bildlichkeit der Fotografie}

Besprochen von SUSANNE HOLSCHBACH

Peter Geimer, Theorien der Fotografie zur Einfübrung, Hamburg (Junius) 2009.

Peter Geimer, Bilder aus Versehen. Eine Geschichte fotografischer Erscheinungen, Hamburg (Philo Fine Arts) 2010.

Bernd Stiegler, Montagen des Realen. Fotografie als Reflexionsmedium und Kulturtechnik, München (Fink) 2009.

Katharina Sykora, Die Tode der Fotografie, Band I, München (Fink) 2009.

Klaus Krüger, Leena Crasemann, Matthias Weiß (Hg.), Um/Ordnungen. Fotografische Menschenbilder zwischen Konstruktion und Destruktion, München (Fink) 2010.

\section{Theorie(n) fotografischer Medialität}

Fotografie ist nach wie vor ein ungeordnetes, umstrittenes Feld: Mit dieser Diagnose beschließt Sabine T. Kriebel ihre Übersichtsdarstellung zu "Theories of Photography», nicht ohne zu betonten, dass genau darin ihr Potenzial liege. Zu einer vergleichbaren Bewertung kommt Peter Geimer in seiner Einleitung zu Ordnungen der Sichtbarkeit, wenn er auf den Vorteil der institutionellen Heimatlosigkeit der Fotografie verweist: «Das Fehlen einer zuständigen Leitdisziplin hält das Feld offen.»² Ein solches Feld übt im akademischen Bereich eine große Anziehungskraft aus - noch scheinen nicht alle Claims abgesteckt, nicht alle Bereiche erschlossen, Entdeckungen nicht unwahrscheinlich. Sicher ist auch damit zu erklären, dass das Interesse an «fotografischen` Themen als Gegenstand von Forschung und Lehre in den letzten Jahren stetig angewachsen ist: nicht nur in Studiengängen mit kulturwissenschaftlicher, medien- oder wissenschaftshistorischer
Ausrichtung, sondern vor allem auch innerhalb der traditionellen Disziplinen. So hat die Kunstgeschichte im Zuge der bildwissenschaftlichen Wende den Bereich der wissenschaftlichen Visualisierung für sich entdeckt und ihre Zuständigkeit in die allgemeine visuelle Kultur ausgedehnt; auch innerhalb der Geschichtswissenschaft bezieht man sich seit einigen Jahren auf den Iconic Turn und beginnt in der Fotografie mehr als nur eine zusätzliche Quelle zu sehen. Ob es sich dabei um eine bloße Konjunktur oder um tiefer gehende Einsichten in die Bedeutung des Bildmediums für die unterschiedlichsten Bereiche von Wissenschaft, Kultur und Gesellschaft handelt - der Bedarf nach einem Kompass für eine erste Orientierung in dem unübersichtlichen Terrain ist jedenfalls groß.

Dem ist inzwischen der Junius Verlag mit der Aufnahme der Theorien der Fotografie in sein Programm Zur Einführung nachgekommen, was nicht zuletzt ein Beleg dafür ist, dass deren Kenntnis neben postkolonialen Theorien, Theorien der Gemeinschaft oder des Internet - um einige andere für dieses Jahr angekündigte Bände der Reihe zu nennen - nun definitiv zu den must haves im geisteswissenschaftlichen Diskurs zu zählen sind.

Geht man von der gesellschaftlichen Bedeutung des Mediums aus, ist man allerdings erneut verwundert, dass es sich dabei tatsächlich um die erste deutschsprachige Überblicksdarstellung dieser Art handelt - abgesehen von Bernd Stieglers ebenfalls erst vor vier Jahren erschienener, eher paraphrasierender Theoriegeschichte der Fotografie. ${ }^{3}$ Maßgeblich für die Ausbildung eines Kanons fototheoretischer Texte bzw. Äußerungen, das belegen auch Stieglers Theoriegeschichte und eine kürzlich von ihm herausgegebene Textsammlung bei Reclam ${ }^{4}$, war Wolfgang Kemps 1980 erschienene Anthologie Theorie der Fotografie I-III, die 2000 mit einem vierten Band von Hubertus von Amelunxen 
ergänzt wurde. ${ }^{5}$ Mit seinen instruktiven Vorworten zu den einzelnen Textauszügen lieferte Kemp eine historische Kontextualisierung; seine Einleitungen, in denen er zwischen Ansätzen, die Fotografie als Kunst, als Medium, als Sprache, als Selbstausdruck oder als Realismus adressieren, unterschied, gaben einem Feld, das sich aus Schriften äußerst heterogener Provenienz konstituierte - Beschreibungen von Erfindern, Kritiken von Literaten, Künstlertexte, Programmschriften von Fotografen, soziologische Studien, semiotische Analysen u.v.a. -, eine erste Struktur. Die heterogenen Bezugsrahmen fotografietheoretischer Reflexionen, die sich, abgesehen von wenigen systematischen Abhandlungen, vielfach in «beiläufigen Bemerkungen" finden, ist eine der Ursachen des ungewissen theoretischen Status der Fotografie, den Geimer in der Einleitung seiner Einführung zu bedenken gibt. Dieser äußert sich bereits in der Unschärfe des Begriffs Fotografie selbst, der sich - im Grunde vergleichbar mit dem Begriff Medium und mit ähnlichen Problemen behaftet - zum einen auf technische Verfahren und deren Produkte, die Bilder, zum anderen auf ein Abstraktum bezieht - ein Abstraktum, das man im Anschluss an Rosalind Krauss auch als das Fotografische bezeichnen kann. ${ }^{6}$ Geimer fokussiert seine Einführung, soweit dies möglich ist, auf das Abstraktum und damit auf die Reflexion und Konzeptualisierung der spezifischen Eigenschaften der Fotografie, d.h. dessen, was sie von anderen Medien und Künsten unterscheidet. Bis auf wenige Ausnahmen - hier wäre vor allem Georges Didi-Huberman zu nennen, dessen Arbeiten eine zentrale Referenz für Geimers Fotografieverständnis darstellen ${ }^{7}$ - entsprechen die besprochenen Autoren und Theoreme denen der Anthologie Kemps/Amelunxens. Der Gewinn liegt vor allem in der sinnvollen Reduktion und der kompakten, gut lesbaren Darstellung, die nicht chronologisch oder nach Autoren, sondern entlang verschiedener Dimensionen der Fotografie vorgeht. ${ }^{8}$ Dadurch wird deutlich, dass die Reflexion auf das Fotografische von Leitmotiven bestimmt wird, die quer zu ihrer mediengeschichtlichen Entwicklung gleichsam insistieren - etwa das Moment der physikalischen Einschreibung, das als Abdruck oder Spur umschrieben, zeichentheoretisch als Index bestimmt oder emphatisch als Emanation des Referenten metaphorisiert wurde (vgl. das erste Kapitel, «Bilder durch Berührung: Fotografie als Abdruck, Spur und Index»), auch wenn diese unterschiedlich gerahmt und zu divergierenden Bewertungen des Mediums eingesetzt wurden bzw. werden.
«While there has been a great deal of focus on the social, political, cultural, and psychological resonances of the photographic medium, it does seem that the actual physical characteristics of the medium and how they signify have gotten short shrift", konstatiert Kriebel in Photography Theory. ${ }^{9}$ Der Bereich fotografischer Materialität liegt zumindest im deutschsprachigen Raum nicht mehr in völligem Dunkel, etwa dank medienarchäologischer Studien, denen auch Geimers ebenfalls in diesem Jahr veröffentlichte Habilitationsschrift Bilder aus Versehen zuzurechnen ist. Aus Versehen deshalb, weil es in Geimers Buch um Unfälle, Zufälle oder Störungen im fotografischen Verfahren geht; Bilder deshalb, weil diese dennoch bzw. gerade dadurch etwas in Erscheinung bringen: die chemisch-physikalische Materialität des Mediums, den Bildträger, die Prozessualität einer Sichtbarmachung. An dieser «verborgenen» Geschichte jenseits der «Erfolgsgeschichte fotografischer Repräsentationen» (S. 54), die Geimer in fotografischen Handbüchern, wissenschaftlichen und parawissenschaftlichen Versuchsanordnungen und Experimenten der künstlerischen Avantgarde auffindet, werden theoretische Grundfragen fotografischer Medialität - die Referenzialität der Fotografie, ihr unklarer Status zwischen 'Erfindung) und (Entdeckung), ihr Bezug zur Kontingenz - erneut verhandelt: insofern kann man die Geschichte fotografischer Erscheinungen auch als Vertiefung und Erweiterung der Einführung lesen. Die meisten der Fallbeispiele sind - zumindest in Expertenkreisen - bekannt; ${ }^{10}$ aufschlussreich sind vor allem Geimers Kontextualisierungen, die Fragen, die er aufwirft, und sein Geschick, vermeintlich bekannte Phänomene begrifflich noch einmal anders zu fassen und dadurch zu leeren Formeln erstarrte Zuschreibungen wieder aufzubrechen. So lassen sich etwa die in fototechnischen Handbüchern als «Feinde der Fotografen d deklarierten Probleme wie das «Abschmelzen der Emulsion», die «blasige Schichtablösung» 9) oder die «Fremdlichteinwirkung» im Sinne Bruno Latours als Akteure begreifen, die die fotografische Forschung erst vorantrieben; erst ihr Potenzial zur «Hervorbringung unvorhersehbarer Aufzeichnungen» (S.349, Hervorh. S.H.), das in wissenschaftlichen Versuchsanordnungen gezielt eingesetzt wurde, definiert die explorative Funktion der Fotografie, indem diese, mit Hans-Jörg Rheinberger gesprochen, «das «Auftreten von Dingen und Zusammenhängen, nach denen man nicht gesucht hat, ermöglicht» (S. 350) - das heißt, ihr Potenzial liegt in dem, was zugleich die "partielle Un- 
verfügbarkeit der Fotografie» (Einführung, S. 68) ausmacht. Vor diesem Hintergrund problematisiert Geimer in einem close reading von Schriften fotografierender Forscher und anhand von Bildbeispielen immer wieder Kategorisierungen und Begrifflichkeiten, die inzwischen zu Allgemeinplätzen geworden sind, wie beispielsweise die «Fotografie des Unsichtbaren» im Zusammenhang mit (nicht nur) der wissenschaftlichen Visualisierung, oder die Metapher des künstlichen Auges, die er als rhetorischen Versuch analysiert, den Bruch zwischen menschlicher Wahrnehmung und dem fotografisch Hergestellten zu kitten.

Dass pauschale Definitionen der fotografischen Me-

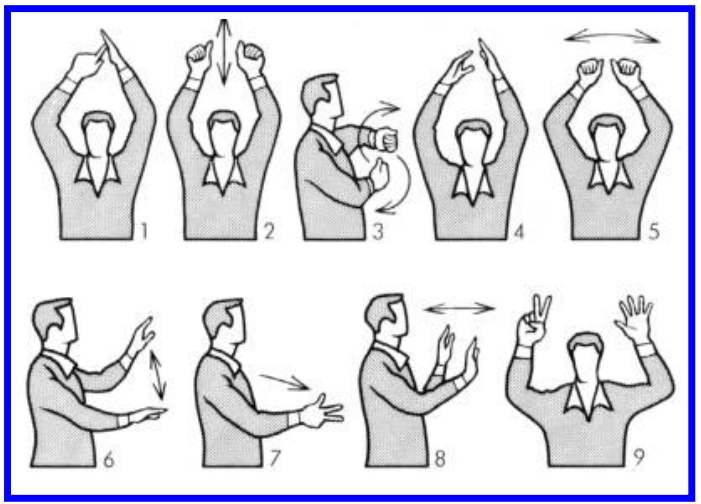

dialität durch Forschungsarbeiten zu unterschiedlichen Gebrauchsweisen, Kontexten und Diskursen der Fotografie dekonstruiert werden bzw. wurden, verhindert leider nicht, dass sie an anderer Stelle unvermindert fortgeschrieben werden, wie beispielsweise in Bernd Stieglers Aufsatzsammlung Montagen des Realen. Wie bereits erwähnt, ist der Literaturwissenschaftler in den letzten Jahren durch eine Reihe von Veröffentlichungen in Erscheinung getreten, die einen Überblick, eine Orientierung oder Einführung in den fototheoretischen Bereich geben wollen. ${ }^{11}$ Auch Montagen des Realen zielt auf eine Auseinandersetzung mit der Fotografie im Allgemeinen: Es gehe ihm um eine historische Kritik der Fotografie, so Stiegler in seiner Einleitung, um «eine kritische Geschichte der Photographie, die die Photographie als Reflexionsmedium und Kulturtechnik [so Stieglers Untertitel] ernst nimmt und zugleich ihre Regeln zu bestimmen sucht» (S. 8). Diesen hohen und sehr weit gefassten Anspruch können die Aufsätze, die aus den unterschiedlichen Interessensgebieten des Autors stammen - Fotografie und Fotografietheorie der Moderne, Pressefotografie, Fotografie und Literatur-, jedoch nicht einlösen; im Gegenteil: Gerade in Stieglers Versuchen, seine größtenteils auf Vortragsmanuskripten und bereits veröffentlichten Aufsätzen beruhenden Einzelbeiträge (das erfährt man allerdings erst aus dem Textnachweis im Anhang) auf einer theoretischen Ebene zu verknüpfen, treten die begrifflichen Unschärfen besonders deutlich zu Tage. So heißt es etwa in einer Passage:

Die Photographie konstituiert das Reale und sie konstituiert es als ein Medium. [...] Die Photographie ist das technische Medium des Realismus. [...] Dieser "Realismus» der Photographie ist seinerseits medial vermittelt, in eigentümlicher Art und Weise gebrochen und, genauer, wird in den Bildern visualisiert und reflektiert. In den Photographien wird sichtbar, was jeweils als Realität verstanden wird: Photographien konstruieren Formen einer angenommenen Wahrheit des Sichtbaren. Photographien sind mediale Konstruktionen der Wirklichkeit. Die Photographie ist ein SehenWollen der Wirklichkeit, eine Materialisierung von bestimmten Wirklichkeitsformen in Bildern. (S. 23f.)

Hier wird einfach Behauptung an Behauptung gereiht, ohne das geklärt würde, ob mit «das Reale» etwas anderes gemeint ist als mit "Wirklichkeit» oder "Realität», ob «Realismus» gleichbedeutend ist mit «Wahrheit des Sichtbaren», was «konstituieren» von «konstruieren» unterscheidet, wann man von "visualisieren" und wann von «reflektieren" sprechen sollte usf. Trotz rhetorischer Anleihen beim Konstruktivismus - «[Es] geht bei der Photographie nicht um Mimesis, sondern um die Mimesis der Mimesis, um eine reflektierte Darstellung der Darstellung, um ein Bild des Bildes der Wirklichkeit» (S. 25) - oder beim performative turn, wenn etwa Gurskys Arbeiten als "Performative des Realen" bezeichnet werden (S. 105), ist eines Stiegler zufolge ganz sicher: "Als Medium des Realismus bleibt die Photographie immer mit der Wirklichkeit verknüpft und kann, was auch immer sie unternimmt, nicht von ihr abgelöst werden». (S. 27) Auch die aktuellen Veränderungen, die die Fotografie in unterschiedlichen medialen Kontexten erfahren habe, ändere daran nichts, so Stiegler an anderer Stelle:

Photographien sind weiterhin visuelle Reflexionen über Realität, sind medial vermittelter und in Bildern konzentrierter Realismus - auch wenn die Realität eine radikal konstruierte ist und mitunter aus nichts anderem besteht als aus am Computer generiertem und bearbeitetem Bildmaterial. (S. 24) 
Solche Nivellierungen und begrifflichen Verunklärungen sind vor allem deswegen so ärgerlich, weil Stiegler damit weit hinter den Stand einer Fotografieforschung zurückfällt, die seit etlichen Jahren genaue Unterscheidungen zu treffen weiß: zwischen fiktionalen und dokumentarischen Strategien der Fotografie, zwischen Bildbearbeitung im Dienste eines künstlerischen Realismus oder einer politischen Manipulation, zwischen Visualisierung und Aufzeichnung, zwischen digital aufgenommenen und am Computer generierten Bildern, um nur einige der offensichtlichsten zu nennen.

\section{Pluralität der Kontexte, Pluralität der Bilder}

Die Aufmerksamkeit auf die Unterschiede zwischen den vielfältigen fotografischen Praktiken gelenkt zu haben, ist vor allem das Verdienst von Positionen, die in kritischer Abgrenzung zu medienontologischen Theorien die fotografische Medialität ausschließlich in den konkreten sozialen Gebrauchsweisen und kulturellen Kodierungen von Fotografien verortet haben - in Geimers Einführung, dargestellt im zweiten Kapitel, «Fotografie als Botschaft und Konstrukt». Diese im weitesten Sinne soziologischen, marxistischen, feministischen, semiotischen oder diskursanalytischen Ansätze haben vor allem seit den 1980er Jahren der Auseinandersetzung mit Fotografie entscheidende Impulse gegeben, indem sie das Feld für Forschungen jenseits von Biografien und Werken fotografischer AutorInnen oder traditioneller Genres geöffnet haben - wie etwa zur kriminalistischen, medizinischen oder ethnografischen Fotografie. ${ }^{12}$ Ein Beispiel für eine aktuelle Veröffentlichung, die auf Arbeiten aus diesem Umfeld aufbaut, ist der erste Band von Die Tode der Fotografie der Kunstwissenschaftlerin Katharina Sykora - das Teilergebnis einer umfangreichen Forschungsarbeit, die sich aus einem Projekt zu theoretischen, medialen und motivgeschichtlichen Fragen nach den "Grenzen der Fotografie»" entwickelt hat. Sykora schließt an Pierre Bourdieus einflussreiche Studie zur Fotografie als Ausdruck und Symptom sozialer Beziehungen an, indem sie unterschiedliche Formen von «Totenfotografie» - Inszenierungen von Toten als Lebende, Aufbahrungsfotos, Sterbebildchen, Porträts auf Grabsteinen u. a. - in ihrer Funktion der individuellen und kollektiven Sinnstiftung innerhalb westlicher Totenriten analysiert. Anders als Bourdieu und seine Forschergruppe, die ihre Thesen aus Befragungen ableiteten, geht Sykora vom Material aus, d.h., sie entschlüsselt anhand kunsthistorisch geschulter Bildbeschreibungen «den Be- deutungsüberschuss, den es [ein Foto] ungewollt verrät, soweit es an der Symbolik einer Epoche, einer Klasse oder einer Künstlergruppe partizipiert». ${ }^{14}$ Sykora erweitert und differenziert auf diese Weise kulturanthropologische Untersuchungen der Regulierung des symbolischen Austausches zwischen den Lebenden und den Toten und deren historischer Veränderung. Darüber hinaus wird durch zahlreiche ganzseitige und farbige Reproduktionen in sehr guter Qualität eine zunehmend verborgene und schließlich verschwundene - zum Teil sehr befremdliche-Bildpraxis wieder sichtbar gemacht: Darüber, ob der 'Trauerrand) des Buches, der durch die schwarze Grundierung der Reproduktionen entstanden ist, nicht etwas über das Ziel hinausschießt, kann man sicher geteilter Meinung sein.

Neben der Totenfotografie im familialen und kriminologischen Kontext behandelt Sykora auch Aufnahmen, die im öffentlichen Raum zirkulier(t)en, beispielsweise Totenbett- bzw. Aufbahrungsfotos von berühmten Staatsmännern oder Hinrichtungsfotos von Staatsgegnern, die durch einen räumlichen Transfer über die Staatsgrenze zu Märtyrerbildern werden können: Denn einmal in Umlauf gebracht, lässt sich der Gebrauch von Fotos nicht mehr im Sinne der Produzenten bzw. ihrer Auftraggeber kontrollieren.

Die wechselvolle Rezeptionsgeschichte solcher Aufnahmen rückt eine Dimension der Fotografie ins Blickfeld, die neben ihrer automatischen Genese ebenfalls von Beginn an als Konstitutiv(um) ihrer spezifischen Medialität reflektiert wurde: ihr Auftreten «im Plural». Unter diesen Begriff stellt die Einführung die Serialität, Reproduzierbarkeit und Zirkulation fotografischer Bilder. Neben prominenten kulturkritischen Positionen zur massenmedialen Wirksamkeit der Fotografie und Benjamins Hypothesen aus seinem "Kunstwerkaufsatz " werden in dem entsprechenden Kapitel auch die im medienwissenschaftlichen Kontext vermutlich weit weniger bekannten Überlegungen André Malraux' vorgestellt, die der französische Essayist und Kulturminister 1947 unter der Überschrift Das imaginäre Museum publiziert hat. Für Malraux ist der Verlust an Materialität bei abfotografierten Artefakten verbunden mit einem Gewinn an Bedeutung: In den «Ver-

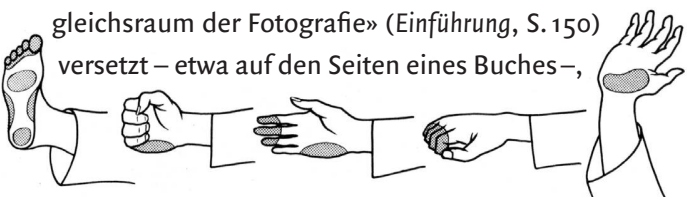


stellen sich Bezüge zwischen einzelnen Werken her, die beispielsweise eine Kategorie wie ‘Stilı als visuellen Effekt erst hervorbringen. ${ }^{15}$

Schon im 19. Jahrhundert wurden massenhaft auch Menschen in den "Vergleichsraum der Fotografie» gestellt: in Porträtalben, im medizinischen, kriminalistischen und anthropologischen Kontext. Arbeiten zu diesen Zusammenhängen bilden die Voraussetzung für das von den Kunsthistorikern Klaus Krüger und Matthias Weiß initiierte Forschungsprojekt Die Performativität fotografischer Menschenbilder. Strategien der Erfassung, Formung und Einverleibung. ${ }^{16}$ Der Sammelband Um/Ordnungen. Fotografische Menschenbilder zwischen Konstruktion und Destruktion stellt die beiden Themenschwerpunkte des Projekts, die «postkoloniale Bildkritik in der künstlerischen Fotografie der Gegenwart" und "Strategien normativer Fotografien aus der Zeit des Nationalsozialismus» in einen größeren Zusammenhang, indem er neben den kunstwissenschaftlichen auch Beiträge von Kultur-, Film- und LiteraturwissenschaftlerInnen sowie von HistorikerInnen einbezieht. Dabei werden zwar Verbindungen zwischen den beiden Schwerpunkten des Projekts hergestellt - in erster Linie durch die Aufsätze zur kolonialen Bildproduktion -, eine Vermittlung der unterschiedlichen Forschungsperspektiven der Beiträger findet jedoch nicht statt, weder durch eine thematische Untergliederung des Bandes noch durch eine fundierte Einführung. So hätte man sich zumindest eine kurze Erläuterung dazu gewünscht, auf welche Forschungsarbeiten sich das Projekt beruft, wie diese weitergeführt werden und welche Erkenntnisse oder Fragestellungen sich aus den Aufsätzen konkret in Bezug auf die Ausgangsthese des Projekts, dass Bilder im generativen Sinne an kulturellen Wandlungsprozessen teilhaben und gestaltend auf sie einwirken (vgl. Vorwort, S. 7), gewinnen lassen.

Das eigentliche Problem dieser und vergleichbarer Textsammlungen liegt jedoch darin, dass das Kriterium «fotografische Medialität» viel zu allgemein ist, um divergierende Forschungsinteressen im Sinne einer so weit gefassten Thematik noch zusammenzuhalten bzw. -zuführen. Dass es trotzdem versucht wird, liegt vielleicht auch daran, dass zu wenig zur Kenntnis genommen wird, wie sehr sich zum einen die Forschung zur Fotografie inzwischen ausdifferenziert hat und zum anderen, dass ihre Transdisziplinarität es im Grunde unmöglich macht, diese noch zu überblicken.
1 Sabine T. Kriebel, Theories of Photography. A Short History, in: James Elkins (Hg.), Photography Theory, New York, London (Routledge) 2007, 42 (siehe dazu auch Anm. 8).

2 Peter Geimer (Hg.), Ordnungen der Sichtbarkeit. Fotografie in Wissenschaft, Kunst und Technologie, Frankfurt/M. (Suhrkamp) 2002, 15 f. An dem institutionellen Nomadentum der Fotografie hat sich seitdem prinzipiell nichts geändert: Neben dem nach wie vor einzigen, aus einer Stiftung hervorgegangenen Lehrstuhl für Theorie und Geschichte der Fotografie - vor Kurzem vom Universitätsverbund Duisburg-Essen an das kunsthistorische Institut der Universität zu Köln übergesiedelt weisen nach wie vor in erster Linie Professorlnnen für Kunstgeschichte und Literaturwissenschaften einen Schwerpunkt in diesem Gebiet aus.

3 Bernd Stiegler, Theoriegeschichte der Fotografie, München (Fink) 2006.

4 Bernd Stiegler (Hg.), Texte zur Theorie der Fotografie, Stuttgart (Reclam) 2010.

5 Wolfgang Kemp (Hg.), Theorie der Fotografie, Bd. I-III, München (Schirmer/Mosel) 1979-1983; Hubertus von Ameluxen ( $\mathrm{Hg}$.), Theorie der Fotografie, Bd. IV: 1980-1995, München (Schirmer/Mosel) 2000.

6 Vgl. Rosalind Krauss, Das Photographische. Eine Theorie der Abstände, München (Fink) 1998.

7 Georges Didi-Huberman hat mit seinen Arbeiten nicht nur neue Forschungsfelder der Fotografie erschlossen (etwa die der Ikonografie der Hysterie), sondern auch theoretische Debatten neu eröffnet hat, wie zuletzt mit Bilder trotz allem, München (Fink) 2007 (zu den vier einzigen Fotografien aus Auschwitz) zur Zeugenschaft der Fotografie.

8 Der Gewinn dieser Vorgehensweise zeigt sich auch im Vergleich mit Kriebels Einführung in «Theories of Photography" (Anm.1), die chronologisch und entlang mediengeschichtlicher Zäsuren vorgeht. Im Unterschied zur deutschen Einführung adressiert das Buch, das neben dem Überblick ein Gespräch und zahlreiche Kurzbeiträge von ExpertInnen zu verschiedenen Aspekten des Themas und zwei weitere Aufsätze von FotografiehistorikerInnen beinhaltet, primär LeserInnen aus dem kunstwissenschaftlichen
Kontext (es ist der zweite Band der Reihe The Art Seminar).

9 Kriebel, Theories, 42.

10 An dieser Stelle sei auch auf eine Veröffentlichung zum Thema "gescheiterter Fotografien « hingewiesen, der es im Unterschied zu der Geimers jedoch primär um die Bilder, nicht um Diskurse geht. Auch wegen der guten Reproduktionen empfiehlt sich der kleine Band als Ergänzung zu Bilder aus Versehen. Clément Chéroux, Fautographie. Petite histoire de l'erreur photographique, Crisnée (Éditions Yellow Now) 2003.

11 Siehe Anm. 3 und 4. Zu nennen wäre außerdem Stiegler, Ein Album fotografischer Metaphern, Frankfurt/M. (Suhrkamp) 2006.

12 Siehe dazu Herta Wolf (Hg.), Diskurse der Fotografie. Fotokritik am Ende des fotografischen Zeitalters, Bd. 2, Frankfurt/M. (Suhrkamp) 2003.

13 Der zweite, noch nicht erschienene Band beschäftigt sich mit Fototheorien, die Tod und Fotografie verknüpfen, und mit künstlerischer Fotografie zum Thema. Zum Forschungsprojekt siehe http://www. hbk-bs.de/hochschule/personen/katharina-sykora/forschungsprojekte/index.php, gesehen am 20.7.2010.

14 Bourdieu, zit. n. Geimer, Einführung, 74 .

15 Abgesehen von einem Verweis auf das Kapitel «Museum ohne Wände" in der Anthologie Paradigma der Fotografie (in dem so überschriebenen Kapitel werden verschiedene Texte abgedruckt, die sich u. a. mit Malraux' Überlegungen in Bezug auf unterschiedliche Kontexte auseinandersetzen in Herta Wolf [Hg.], Paradigma der Fotografie. Fotokritik am Ende des fotografischen Zeitalters, Frankfurt/M. [Suhrkamp] 2002, 349-457) hätte sich hier auch eine Auseinandersetzung mit dem Bilderatlas Aby Warburgs anschließen lassen, dessen epistemisches Potenzial in den letzten Jahren in unterschiedlichen Zusammenhängen diskutiert wurde; vgl. z. B. Sabine Flach, Inge Münz-Koenen, Marianne Streisand (Hg.), Der Bilderatlas im Wechsel von Künsten und Medien, München (Fink) 2005.

16 Das Projekt ist Teil des Sonderforschungsbereichs Kulturen des Performativen an der FU Berlin, siehe http:||www2.hu-berlin.de/performativ| seiten/a8.html, gesehen am 2.08.10. 